\title{
Cassiopeia A dust composition and heating ${ }^{\star}$
}

\author{
T. Douvion ${ }^{1,2}$, P. O. Lagage ${ }^{1}$, and E. Pantin ${ }^{1}$ \\ 1 DSM/DAPNIA/Service d'Astrophysique, CEA/Saclay, 91191 Gif-sur-Yvette, France \\ ${ }^{2}$ Supélec, Service des Mesures, 91192 Gif-sur-Yvette, France
}

Received 28 July 2000 / Accepted 9 January 2001

\begin{abstract}
Material ejected by a supernova is directly observed in the form of Fast Moving Knots (FMK's) in the Cassiopeia A supernova remnant. Part of this material has condensed into dust. In this paper, by fitting the ISO mid-infrared emission of the dust, we derive the dust composition and discuss the dust heating. The dust is found to be made mainly of three components: pyroxene, quartz and aluminium oxide. These components have to be heated at two different temperatures, a "hot" temperature around $350 \mathrm{~K}$ and a cold temperature around $90 \mathrm{~K}$. The physical conditions in the knot-shocked region are such that the dust can be collisionally heated to a temperature of $350 \mathrm{~K}$. The cold dust temperature can also be well accounted for by the knot model discussed in the paper. The dust formation efficiency is tentatively estimated to be of the order of $10 \%$ in this supernova.
\end{abstract}

Key words. supernova remnant - supernovae individual: Cas A dust - infrared: general

\section{Introduction}

Cassiopeia A (Cas A) is the youngest SuperNova Remnant (SNR) known in our galaxy. The date of the SN explosion which has given birth to the SNR is estimated to be 1680 (Fesen et al. 1988). The SN was subluminous and/or heavely obscured, since it was not noticed, except perhaps by Flamsteed (Ashworth 1980). The progenitor of the SN was a massive star (Vink et al. 1996; Jansen et al. 1988; Fabian et al. 1980), probably of Wolf-Rayet type (Fesen et al. 1988). Freshly synthesized SN material is observed in the form of Fast Moving Knots (FMK's) (Baade \& Minkowsky 1954; Chevalier \& Kirshner 1979; van den Bergh \& Kamper 1985 and earlier papers). There are several evidences indicating that part of the SN ejecta has condensed into dust (Lagage et al. 1996; Arendt et al. 1999, hereafter ADM99, Douvion et al. 1999, hereafter DLC99). In this paper, after having presented in Sect. 2 the observational material obtained with the Infrared Space Observatory (ISO) (Kessler et al. 1996), we discuss, in Sect. 3, the dust composition and, in Sect. 4, the dust heating.

Send offprint requests to: T. Douvion, e-mail: Thomas.Douvion@supelec.fr or lagage@cea.fr

* Based on observations with ISO, an ESA project with instruments funded by ESA Member States (especially the PI countries: France, Germany, The Netherlands and the UK) and with the participation of ISAS and NASA, and on observations obtained at Canada-France-Hawaii Telescope.

\section{Observations}

\subsection{ISO-SWS observations}

The mid-InfraRed (IR) wavelength range is rich with dust features, especially silicate features. The Short Wavelength Spectrometer (SWS) on board of ISO (de Graauw et al. 1996) has been a powerful instrument to reveal and study dust features (e.g. Waelkens et al. 1999). The SWS observation of Cas A, discussed here, was performed on December 5th 1997. The observation consists of a low-resolution full grating scan from 2.38 to $45.2 \mu \mathrm{m}$. The position on the sky of the SWS slit is shown in Fig. 1. The data reduction was first performed using the Observer SWS Interactive Analysis (OSIA ${ }^{1}$ ) package. Given that the Cas A flux is close to SWS sensitivity limit, additional data reduction was needed. Indeed, the data scans are corrupted with random "jumps" in the signal, originating probably from high energy particles hitting the detectors; these "jumps" may create long term shifts in the signal and degrade seriously the quality of the dark. To deal with the jump problem, we have used a package developed under SIA ${ }^{1}$ (Spectroscopic Interactive Analysis) by Pantin et al. (1999). Even with this careful data reduction, the signal to noise ratio is high enough only in the 16-29 $\mu \mathrm{m}$ wavelength range. In this wavelength range, the aperture of the SWS slit is equal to $14^{\prime \prime}$ by $27^{\prime \prime}$. The resulting spectrum is displayed in Fig. 2. The spectrum is similar in shape and flux level to the spectrum N3 discussed

1 OSIA and SIA are joint developments by the ESA astrophysics division, the ISO-SWS, and the ISO-LWS consortium. 


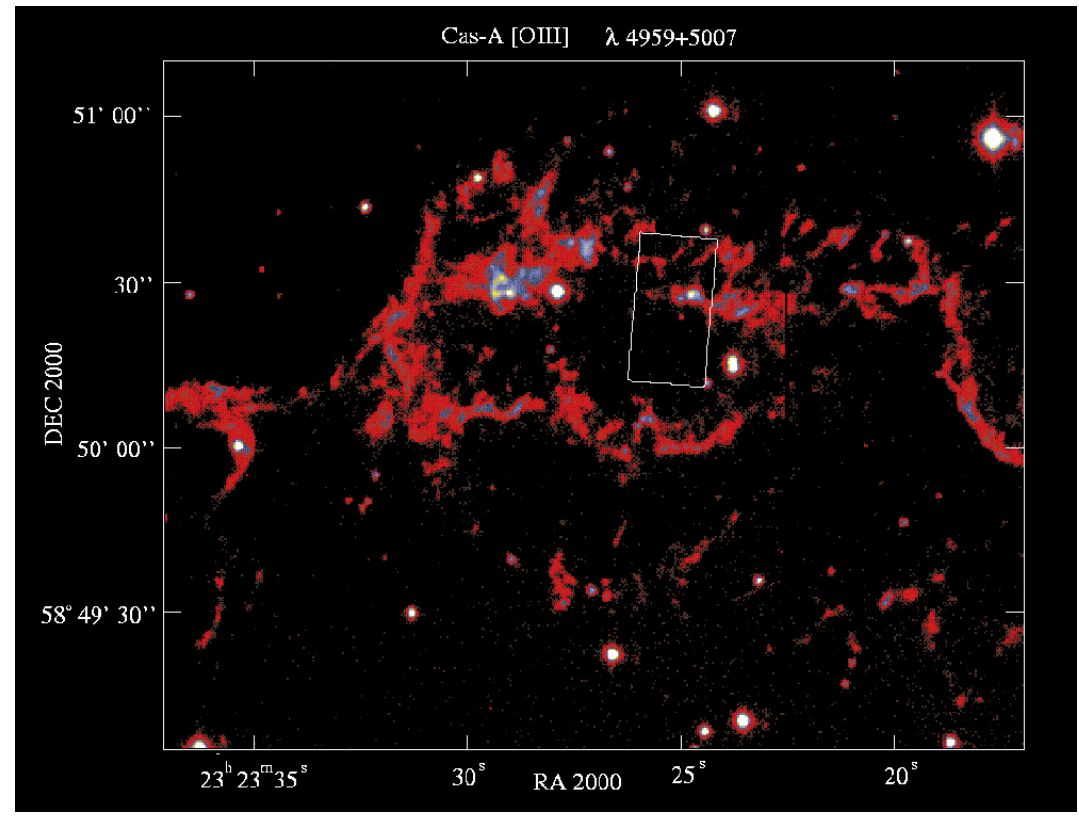

Fig. 1. Optical image of the northern part of the Cas A SNR in the [OIII] lines at 4959 and $5007 \AA$, obtained with the SIS instrument mounted on the Canada France Hawaii Telescope (CFHT) on August 25th 1998. The white rectangle indicates the size and the position of the ISO-SWS slit aperture of the observations discussed in this paper

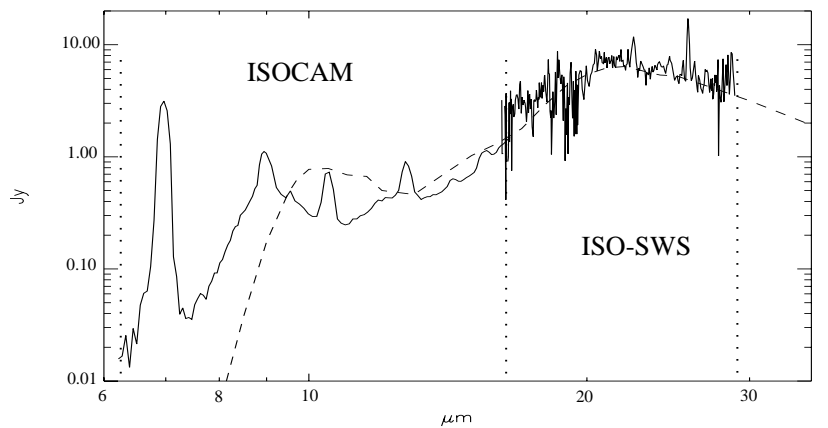

Fig. 2. Full line: ISOCAM $(6-16 \mu \mathrm{m})$ and ISO-SWS (16$29 \mu \mathrm{m}$ ) fluxes obtained in the aperture plotted in Fig. 1, as a function of the wavelength. The uncertainties are of $\pm 6 \mathrm{mJy}$ on CAM data and \pm 1 Jy on SWS data. In addition to the continuum emission that is discussed in the paper, we identify forbidden ionic line emission from [ArII] $(7.0 \mu \mathrm{m})$, [ArIII] $(9.0 \mu \mathrm{m})$, [SIV] $(10.5 \mu \mathrm{m}),[\mathrm{NeII}](12.8 \mu \mathrm{m}),[\mathrm{NeV}](14.3 \mu \mathrm{m}$, very weak), [NeIII] $(15.5 \mu \mathrm{m})$ and [OIII] $(25.9 \mu \mathrm{m})$. The dashed line is the fit of the continuum emission with $\mathrm{Mg}$-protosilicates heated at $169 \mathrm{~K}$, as proposed by ADM99

in ADM99. The slit positions on the sky corresponding to the two spectra were very close, so that, given the pointing uncertainties of ISO, the main emitting area is probably contained in both slit apertures.

\subsection{ISOCAM observations}

The spectrum at wavelengths shorter than $16 \mu \mathrm{m}$ was obtained with ISOCAM, the camera on board of ISO (Cesarsky et al. 1996). The ISOCAM observations were performed on December 5th 1996, with a pixel field of view of $6^{\prime \prime}$ and a corresponding total field of view of $3^{\prime} \times 3^{\prime}$. The field was centered on the northern part of the remnant. For each pixel, we have a spectrum from 6 to $16.5 \mu \mathrm{m}$, obtained by rotating the Circular Variable Filter (CVF) of ISOCAM; the spectral resolution obtained that way is $\lambda / \Delta \lambda \simeq 40$. The data reduction was performed with the Cam Interactive Analysis ${ }^{2}$ (CIA) package. We have reconstructed the 7 to $16 \mu \mathrm{m}$ fluxes in the $14^{\prime \prime}$ by $27^{\prime \prime} \mathrm{SWS}$ aperture, by co-adding the ISOCAM fluxes in this area. The resulting spectrum is shown in Fig. 2. Note that no re-scaling was applied to connect the SWS spectrum and the ISOCAM spectrum.

\section{Dust composition}

Following ADM99, we have first tried to fit the spectrum of Fig. 2 with Mg-protosilicates at a temperature of $169 \mathrm{~K}$; the optical constants of $\mathrm{Mg}$-protosilicates have been taken from Dorschner et al. (1980). The 16-29 $\mu \mathrm{m}$ part of the spectrum is well fitted, but the model predicts a $10 \mu \mathrm{m}$ feature quite different from the observed one (see Fig. 2). Another material has to be found. The choice of materials is large, so that we have used, as a starting point, the materials which are predicted to form in abundance from $\mathrm{SN}$ material, i.e. $\mathrm{MgSiO}_{3}$ (pyroxene) and $\mathrm{Al}_{2} \mathrm{O}_{3}$ (Kozasa et al. 1991; Kozasa \& Hisato 1997).

We first focused on fitting the feature at $9.5 \mu \mathrm{m}$. The use of $\mathrm{MgSiO}_{3}$ leads to a better fit of the feature than Mg-protosilicates; however, the predicted flux is too low in the $7.5-8.5 \mu \mathrm{m}$ range (see Fig. 3). Whatever the various silicates we use, (olivine or pyroxene with various $\mathrm{Mg} / \mathrm{Fe}$ ratio, Dorschner et al. 1995, various protosilicates, Dorschner et al. 1980, Draine and Lee astronomical silicates, Draine \& Lee 1984), whatever the dust temperature is, the problem persists. The use of $\mathrm{Al}_{2} \mathrm{O}_{3}$ is of no help in that region. To solve the problem, we had to introduce another dust component made of quartz $\left(\mathrm{SiO}_{2}\right)$. As can be seen in Fig. 3, a good fit of the $9.5 \mu \mathrm{m}$

\footnotetext{
${ }^{2}$ CIA is a joint development by the ESA astrophysics division and the ISOCAM consortium.
} 


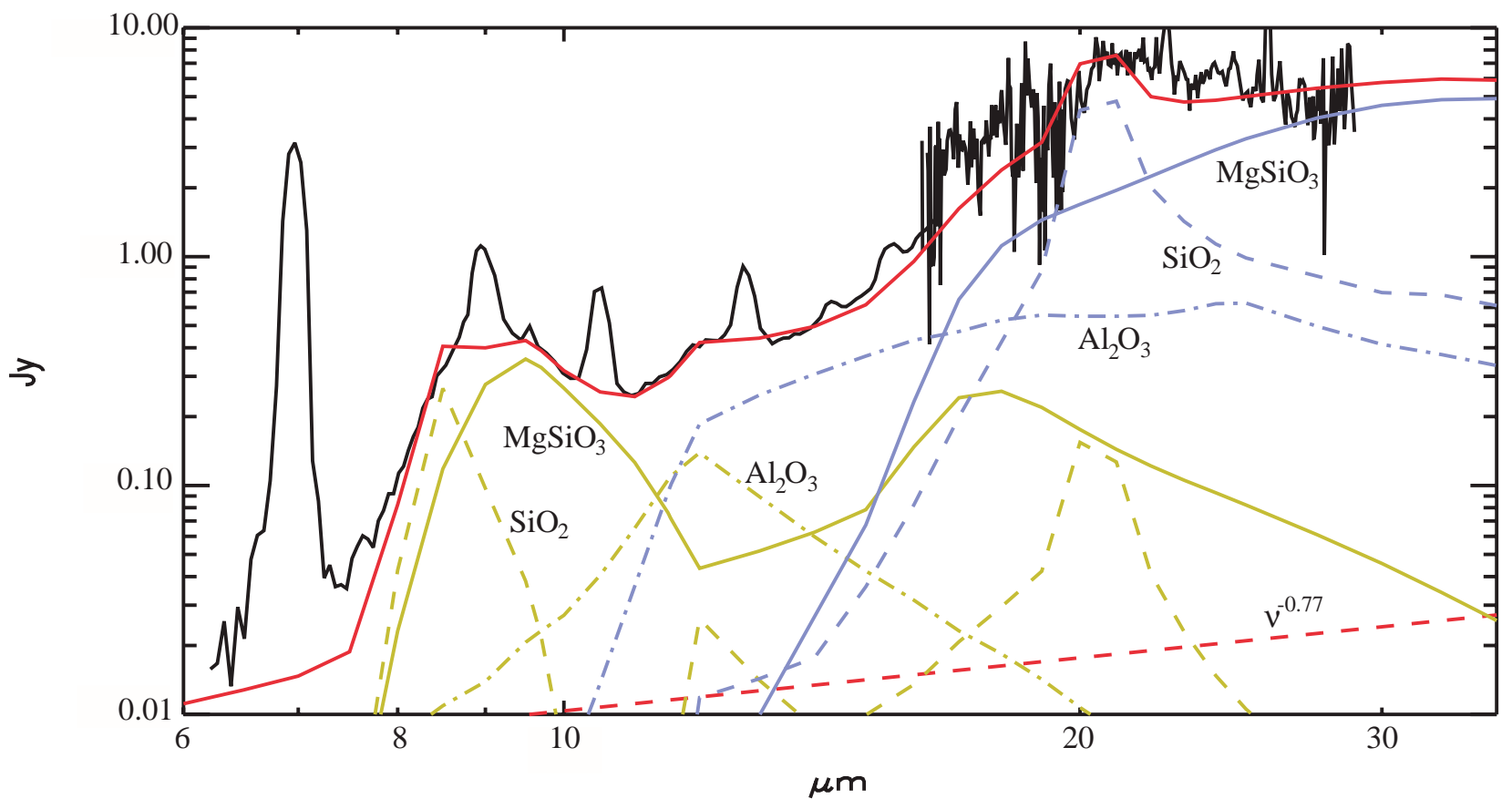

Fig. 3. ISOCAM and ISO-SWS data fitted with pyroxene (full blue and green lines), quartz (dashed blue and green lines) and aluminium oxides (dot-dashed blue and green lines). The hot component is figured in green, and the cold component in blue. The red dashed line is the possible synchrotron continuum contribution (slope from Baars et al. 1977). The red line is the result of the sum of the contributions of the various components

feature is obtained with a contribution of both pyroxene and quartz at a relatively high temperature $(345 \mathrm{~K}$ for pyroxene and $361 \mathrm{~K}$ for quartz); (the optical constants of the materials are obtained from Dorschner et al. 1980 and from the database on the web site of the Jena institute, http://www . astro.uni-jena.de/Group/Subgroups/ Labor/Labor/databases.html). It is not surprising to find quartz here. Indeed, quartz can be produced with a good efficiency from SN material (Kozasa 2000, private communication). In the absence of magnesium, the formation of pyroxene through the reaction $\mathrm{Mg}_{\text {gaz }}+\mathrm{SiO}_{\text {gaz }}+2 \mathrm{O}_{\text {gaz }} \rightarrow \mathrm{MgSiO}_{3}$ solid is quenched and quartz is formed instead. If the element mixing is uncomplete, as claimed in DLC99, then there is indeed a layer inside the $\mathrm{SN}$, with $\mathrm{Si}, \mathrm{O}$ and lack of $\mathrm{Mg}$ (Woosley $\&$ Weaver 1995). In case of complete mixing, given that $\mathrm{Si}$ is more abundant that $\mathrm{Mg}$, the formation of $\mathrm{SiO}_{2}$ is also possible.

The same relative quantities of quartz and pyroxene, but at a cooler temperature (83 K for pyroxene and $93 \mathrm{~K}$ for quartz), lead also to a good fit of the data in the $16-29 \mu \mathrm{m}$ wavelength range. Finally, the presence of aluminium oxides (hot at $351 \mathrm{~K}$ and cold at $113 \mathrm{~K}$ ) accounts for the $12-13 \mu \mathrm{m}$ feature, and contributes dominantly to the continuum from 13 to $16 \mu \mathrm{m} . \mathrm{Al}_{2} \mathrm{O}_{3}$ exists in different forms; the best fit was obtained with a mixture of $\mathrm{Al}_{2} \mathrm{O}_{3}$ called ISAS in the paper by Koike et al. (1995); the mixture mainly consists of $\gamma-\mathrm{Al}_{2} \mathrm{O}_{3}$. Note that we had to decrease by a factor of 5 the relative abundance of $\mathrm{Al}_{2} \mathrm{O}_{3}$ compared to $\mathrm{MgSiO}_{3}$, when going from the hot component to the cold component (see next section). The resulting fit is shown in Fig. 3 .

\section{Dust heating}

In the previous section, we only discussed the dust composition; in this section, we discuss the physical model we have used to account for the dust heating. As pointed out in the paper by Lagage et al. (1996), the dust is probably SN condensates embedded in the FMK's. As discussed by Dwek \& Werner (1981), the dominant dust heating mechanism in the FMK's is collisions between the dust and the electrons of the plasma inside the knots. To calculate the heating of the dust, we need to know the physical conditions inside the FMK's. Constraints on these physical conditions can in principle be obtained from optical observations.

\subsection{Optical fluxes}

There is not yet a model reproducing all the optical observations of a knot. The most developed model is, so far, the model by Sutherland \& Dopita (1995) (SD95 hereafter). The basis of the model is the encounter of a FMK with the reverse shock of the SN, which induces the propagation of a shock wave into the knot, preceeded by the propagation of an ionisation front. Oxygen lines arise both at the ionisation front and at the shock, which becomes rapidly radiative. In the following, we adopt the SD95 model with a shock velocity inside the knot of $V_{\mathrm{s}}=200 \mathrm{~km} \mathrm{~s}^{-1}$. We have chosen this model because it is the one which predicts the right [OIII] $4363 /[\mathrm{OIII}]$ $4959+5007$ line flux ratio. Most of the optical [OIII] lines are produced at the ionisation front, which propagates at a high speed of $3300 \mathrm{~km} \mathrm{~s}^{-1}$. The flux observed in the 
[OIII] 4959+5007 $\AA$ lines allows us to determine the density in this region using the SD95 model. For the bright optical knot contained in the SWS aperture (see Fig. 1), we measure $F_{\text {SingleKnot }}([\mathrm{OIII}])=1.510^{-11} \mathrm{erg} \mathrm{cm}^{-2} \mathrm{~s}^{-1}$ from CFHT observations, taking into account the extinction in the same way as Dinerstein et al. (1987). The electronical temperature, derived from SD95 for the part of the ionisation front where [OIII] is present, is $710^{3} \mathrm{~K}$ and, given the physical parameters of the lines emission deduced from Osterbrock (1989), we obtain a knot electronic density, $n_{\mathrm{e}}$, of $2.410^{3} \mathrm{~cm}^{-3}$. We assume a spherical geometry and we take Cas A at a distance of $3.4 \mathrm{kpc}$ (Reed et al. 1995), and a typical diameter knot size of $1^{\prime \prime}$, (size of the brightest optical knot in the SWS aperture, as deduced after deconvolution of the optical image shown in Fig. 1).

\subsection{Infrared fluxes and dust mass in the hot component}

We consider here the dust heating in the shock cooling region. The electronic temperature of the shocked material decreases rapidly in the cooling region from $T_{\mathrm{e}}=10^{6.64} \mathrm{~K}$ to $T_{\mathrm{e}}=300 \mathrm{~K}$; the associated electronic density varies from $n_{\mathrm{e}}=9.610^{3} \mathrm{~cm}^{-3}$ to $n_{\mathrm{e}}=310^{7} \mathrm{~cm}^{-3}$; (rescaled from SD95). The physical conditions in this region are such that the dust can be collisionally heated at a temperature corresponding to the temperature of the "hot" dust component discussed in the previous section. Thus we have considered that the hot dust component was associated with this cooling region. In order to derive the "hot" dust mass from the IR observations, we need to know the size of the dust. We have no observational constraint on these sizes, so that we have taken the sizes calculated by Kozasa et al. (1997) for newly formed SN dust: $70 \AA$ for the $\mathrm{MgSiO}_{3}$, and $10 \AA$ for the $\mathrm{Al}_{2} \mathrm{O}_{3}$ grains; we have also used $70 \AA$ for the $\mathrm{SiO}_{2}$ grains. With these sizes and given the dust heating, the IR luminosities imply $7.810^{-9} M_{\odot}$ of $\mathrm{MgSiO}_{3}, 3.110^{-9} M_{\odot}$ of $\mathrm{SiO}_{2}$ and $1.110^{-9} M_{\odot}$ of $\mathrm{Al}_{2} \mathrm{O}_{3}$. Note that a $30 \AA$ change in the size of a $70 \AA$ grain leads to a change by a factor 3 of the masses.

Are such masses compatible with dust made from SN material? In DLC99, we have argued that the dust observed from Cas A was silicate dust made from SN material; the layer in the $\mathrm{SN}$ where silicate components ( $\mathrm{Si}, \mathrm{Mg}$ and $\mathrm{O}$ ) are present, was identified in Fig. 2 of DLC99. If we take the relative abundance of elements in the "silicate layer" (Woosley \& Weaver 1995), we can evaluate the mass ratio of the different dust species relatively to the gas. Assuming that the dust formation efficiency is $100 \%$, then almost all the magnesium and part of the silicon is locked in $\mathrm{MgSiO}_{3}$ dust grains; the remaining silicon is locked in $\mathrm{SiO}_{2}$ dust grains; all the aluminium is locked in $\mathrm{Al}_{2} \mathrm{O}_{3}$ dust grains, and the remaining gas is almost entirely made of oxygen. In these conditions, $M_{\mathrm{MgSiO}_{3}} / M_{\mathrm{gas}}=0.40, M_{\mathrm{SiO}_{2}} / M_{\mathrm{gas}}=0.14$ and $M_{\mathrm{Al}_{2} \mathrm{O}_{3}} / M_{\text {gas }}=0.032$. The size of the cooling region, and the density derived from CFHT observations allow to calculate the mass of gas available in the cooling area. This region is very thin; SD95 obtained a cooling length, $l_{\text {cool }}$, of $210^{11} \mathrm{~cm}$ for an initial oxygen density of $1.210^{3} \mathrm{~cm}^{-3}$ (here the oxygen density has been taken equal to $n_{\mathrm{e}} / 2$ according to the degree of oxygen ionization calculated in SD95 in the ionization area). Then the mass of gas available in the cooling region of a single knot is $1.310^{-7} M_{\odot}$; consequently, if we consider a dust formation efficiency of $100 \%$, we obtain $M_{\mathrm{MgSiO}_{3}}$ $=5.310^{-8} M_{\odot}, M_{\mathrm{SiO}_{2}}=1.810^{-8} M_{\odot}$ and $M_{\mathrm{Al}_{2} \mathrm{O}_{3}}=$ $4.310^{-9} M_{\odot}$. Thus the observed and potentially available dust masses are compatible.

Comparing the observed dust masses and the calculated maximum dust mass produced from SN material in a knot, we can evaluate the dust "formation" efficiency. When the SWS aperture contains many knots, a map obtained at $9.77 \mu \mathrm{m}$ with ISOCAM using the smallest pixel field of view of $1.5^{\prime \prime}$, shows that the hot component originates mainly $(50 \%)$ from the bright knot of the SWS aperture of Fig. 1. For this knot, we can derive an estimate of dust formation efficiency of $e_{\mathrm{MgSiO}_{3}}=7.4 \%, e_{\mathrm{SiO}_{2}}=8.6 \%$ and $e_{\mathrm{Al}_{2} \mathrm{O}_{3}}=13 \%$. Given the simplifications in the model and the uncertainties in the parameters of the region (a factor 2 of error on the initial density leads to a factor 2 of error on the efficiency), we can just state that the efficiency is of the order of $10 \%$. Note that the observed $M_{\mathrm{MgSiO}_{3}} / M_{\mathrm{SiO}_{2}}$ and $M_{\mathrm{MgSiO}_{3}} / M_{\mathrm{Al}_{2} \mathrm{O}_{3}}$ ratios are well predicted by the model.

\subsection{Infrared fluxes and dust mass in the cold component}

Much more dust is present in the region behind the cooling region. From the SD95 model, we can deduce that the "mean" physical conditions in that region are $n_{\mathrm{e}}=310^{7} \mathrm{~cm}^{-3}$ and $T_{\mathrm{e}}=300 \mathrm{~K}$. Given these physical conditions and assuming the same dust sizes as for the "hot" component, we can compute the dust temperature and deduce from the observed fluxes the "cold" dust masses needed: $M_{\mathrm{MgSiO}_{3}}^{\text {cold }}=1.110^{-4} M_{\odot}, M_{\mathrm{SiO}_{2}}^{\text {cold }}=$ $4.410^{-5} M_{\odot}$ and $M_{\mathrm{Al}_{2} \mathrm{O}_{3}}^{\text {cold }}=3.210^{-6} M_{\odot}$.

The dust mass in the region behind the cooling area is difficult to determine. An upper limit to the dust mass can be obtained by considering the total dust mass in a knot. From the gas to dust mass ratio (DLC99) and the density previously discussed, we can derive a total dust mass of $M_{\mathrm{MgSiO}_{3}}=4.210^{-4} M_{\odot}, M_{\mathrm{SiO}_{2}}=1.510^{-4} M_{\odot}$ and $M_{\mathrm{Al}_{2} \mathrm{O}_{3}}=3.410^{-5} M_{\odot}$, in the brightest knot in the SWS aperture. If we assume, as for the hot component, that half of the cold dust is located into this knot, the observed cold component from the knot represents about $14 \%$ of the maximum $\mathrm{MgSiO}_{3}$ and $\mathrm{SiO}_{2}$ dust masses. We are in the uncomfortable situation that the efficiency deduced for the cold dust is somewhat higher than that deduced from the hot component. The assumption that half 
of the observed cold dust is present in a bright knot, which cannot be tested because of the poor angular resolution of the SWS instrument, is questionable. Indeed the cold component emission lasts longer than the hot component emission, just because of the rapid cooling of region where the dust hot component is emitted and the rapid propagation of the shock inside the knot. Then it is quite possible that several knots with no or little hot dust emission are present in the SWS beam; this would explain why we observe so much dust in the cold component. Note that the $\mathrm{Al}_{2} \mathrm{O}_{3}$ cold component mass is only $5 \%$ of the total expected dust mass. The origin of such a large depletion compared to $\mathrm{MgSiO}_{3}$ or $\mathrm{SiO}_{2}$ may be linked to the small size $(10 \AA)$ of the grains, which are more easily destroyed by sputtering effects in the cooling area than the $70 \AA ̊$ A sized particles.

One consequence of the model presented here is the temporal correlation between the optical emission at the shock and the hot dust emission. We also predict an increase with time of the IR emission from the cold dust component. Monitoring the knots both in the IR and the optical on the time scale of the knot optical lifetime (about 30 years) should help qualifying the model presented here. High angular resolution at $20 \mu \mathrm{m}$ are also needed to assess if there are indeed knots emitting mostly at $20 \mu \mathrm{m}$, with faint counterparts in the optical or at $10 \mu \mathrm{m}$.

Acknowledgements. We thanks A. Jones and J.-P. Chièze for enlightening discussions on shocks and their effects, and D. Péquignot for discussions on the optical emission.

\section{References}

Arendt, R. G., Dwek, E., \& Moseley, S. H. 1999, ApJ, 521, 234 (ADM99)

Ashworth, W. B. 1980, J. Hist. Astron., 11, 1

Baade, W., \& Minkowski, R. 1954, ApJ, 501, 206

Baars, J. W. M., Genzel, R., Pauliny-Toth, I. I. K., \& Witzel, A. 1977, A\&A, 61, 99
Cesarsky, C. J., Abergel, A., Agnese, P., et al. 1996, A\&A, 315, L32

Chevalier, R. A., \& Kirshner, R. P. 1979, ApJ, 233, 154

de Graauw, T., Haser, L. N., Beintema, D. A., et al. 1996, A\&A, 315, L49

Dinerstein, H. L., Lester, D. F., Rank, D. M., et al. 1987, ApJ, 312,314

Dorschner, J., Friedmann, C., Gütler, J., \& Duley, W. W. 1980, A\&ASS, 68, 159

Dorschner, J., Begemann, B., Henning, T., Jäger, C., \& Mutschke, H. 1995, A\&A, 300, 503

Douvion, T., Lagage, P.-O., \& Cesarsky, C. J. 1999, A\&A, 352, L111 (DLC99)

Draine, B. T., \& Lee, H. M. 1984, ApJ, 2885, 89

Dwek, E., \& Werner, M. W. 1981, ApJ, 248, 138

Fabian, A. C., Willingale, R., Pye, J. P, Murray, S. S., \& Fabbiano, G. 1980, MNRAS, 193, 175

Fesen, R. A., Becker, R. H., \& Goodrich, R. W. 1988, ApJ, 329, L89

Jansen, F. A., Smith, A., Bleeker, J. A. M., et al. 1988, ApJ, 331,949

Kessler, M. F., Steinz, J. A., Anderegg, M. E., et al. 1996, A\&A, 315, L27

Koike, C., Kaito, C., Yamamoto, T., et al. 1995, Icarus, 114, 203

Kozasa, T., Hasegawa, H., \& Nomoto, K. 1991, A\&A, 249, 474

Kozasa, T., \& Hisato, S. 1997, APSS, 255, Issue 1/2, 437

Lagage, P. O, Claret, A., Ballet, J., et al. 1996, A\&A, 315, L273

Osterbrock, D. E. 1989, Astrophysics of gaseous nebulae and active galactic nuclei (University Science Books)

Pantin, E., Waelkens, C., \& Malfait, K. 1999, The Universe as seen by ISO, ESA SP-427, 385

Reed, J. E., Hester, J. J., Fabian, A. C., \& Winkler, P. F. 1995, ApJ, 440, 706

Sutherland, R. S., \& Dopita, M. A. 1995, ApJ, 439, 381 (SD95)

van den Bergh, S., \& Kamper, K. 1985, ApJ, 293, 537

Vink, J., Kaastra, J. S., \& Bleeker, J. A. M. 1996, A\&A, 307, L41

Waelkens, C., Malfait, K., Waters, L. B. F. M., \& de Graauw, Th. 1999, The Universe as seen by ISO, ESA SP-427, 607

Woosley, S. E., \& Weaver, T. A. 1995, ApJS, 101, 181 